\title{
Extending the baryon ChPT beyond the low-energy region
}

\section{Jambul Gegelia*}

Institute for Advanced Simulation, Institut fuer Kernphysik and Juelich Center for Hadron

Physics, Forschungszentrum Juelich, D-52425 Juelich, Germany

Tbilisi State University, 0186 Tbilisi, Georgia

E-mail: jgegelia@hotmail.com

\begin{abstract}
We present an extension of the one-nucleon sector of baryon chiral perturbation theory beyond the low-energy region. Applicability of the proposed approach for higher energies is restricted to small scattering angles, i.e. the kinematical region where the quark structure of hadrons cannot be resolved. The main idea is to re-arrange the low-energy effective Lagrangian according to a new power counting and for loop diagrams exploit the freedom of the choice of the renormalization condition. We generalize the extended on-mass-shell scheme for the one-nucleon sector of the baryon chiral perturbation theory by choosing a sliding scale, i.e. expand the physical amplitudes around kinematical points far beyond the threshold. This requires an introduction of complexvalued renormalized coupling constants which can be either extracted from experimental data, or calculated using the renormalization group evolution of coupling constants fixed in threshold region.
\end{abstract}

The 8th International Workshop on Chiral Dynamics

29 June 2015 - 03 July 2015

Pisa, Italy

${ }^{*}$ Speaker. 
The main idea of Effective field theories (EFTs) of the strong interaction, started with Ref. [1], is that by considering the most general effective Lagrangian of the relevant light degrees of freedom, which is invariant under all symmetries of quantum chromodynamics (QCD), one can reproduce the low-energy structure of the $S$-matrix of QCD. Contributions of heavy degrees of freedom are represented by a systematic expansion of the effective Lagrangian in powers of quark masses and derivatives acting on fields. The Goldstone-boson sector of chiral EFT, called chiral perturbation theory (ChPT) [2], turned out to be very successful. The inclusion of nucleons in this framework encountered problems due to the non-vanishing nucleon mass in the chiral limit[3]. The encountered problem of power counting in baryon ChPT (BChPT) has first been resolved by applying the heavy baryon approach $[4,5,6]$. Later it has been observed that the power counting violating parts of loop diagrams in manifestly Lorentz invariant formulations of BChPT are polynomial in quark masses and external momenta and can be subtracted systematically by renormalizing the parameters of the effective Lagrangian [7, 8, 9, 10, 11, 12]. For more details see, e.g., Refs. [13, 14].

Here we present an extention of the applicability of BChPT beyond the low-energy region for small scattering angles (for more details see Ref. [15]). This is achieved by re-arranging the chirally invariant terms of the standard low-energy effective Lagrangian and by introducing a generalization of the extended on-mass-shell (EOMS) scheme of Refs. [10, 11, 12]. We obtain an EFT with new well-defined power counting rules. Loop diagrams contributing to physical amplitudes violate this power counting. However, the divergent parts as well as power counting violating pieces can be subtracted by applying a generalization of the EOMS scheme. The subtracted terms are absorbed in the redefinition of parameters of the re-arranged effective Lagrangian. As the subtractions are made above the threshold, the corresponding counter terms contain imaginary parts. This means that the renormalized parameters become complex. Thus, the suggested modification of the EOMS scheme belongs to the class of complex mass schemes (CMS) first considered in Refs. [16, 17]. One might be concerned about unitarity within the CMS because of the use of complex renormalized parameters, however, this issue has been discussed in detail recently in Ref. [18] (see also Ref. [19]).

Calculations are perturbative in the one-nucleon sector of BChPT within the new approach, i.e. we obtain a finite number of diagrams at any finite order. However this framework can be applied only close to the forward direction, where the quark structure of hadrons cannot be resolved. The branch points and cuts of the $S$-matrix of QCD are generated by loop diagrams in the EFT framework. On the other hand, the poles in the $S$-matrix require the inclusion of the corresponding fields as explicit degrees of freedom in the effective Lagrangian or performing some kinds of non-perturbative resummations. That is, all resonances which appear in the considered region of energies must be included as dynamical degrees of freedom in the effective Lagrangian.

\section{Re-arranging tree-order diagrams and the effective Lagrangian}

We consider the process $\pi^{a}(q) N(p) \rightarrow \pi^{a^{\prime}}\left(q^{\prime}\right) N\left(p^{\prime}\right)$ in isospin-symmetric limit, where $a$ and $a^{\prime}$ are Cartesian isospin indices. We parameterize the pion-nucleon scattering amplitude as [22]

$$
T_{a a^{\prime}}=\delta_{a a^{\prime}} T^{+}+\frac{1}{2}\left[\tau_{a}, \tau_{a^{\prime}}\right] T^{-},
$$




$$
T^{ \pm}=\bar{u}\left(p^{\prime}, \sigma^{\prime}\right)\left[D^{ \pm}(t, v)-\frac{1}{4 m_{N}}\left[d^{\prime}, q\right] B^{ \pm}(t, v)\right] u(p, \sigma) .
$$

In BChPT it is convenient to work with the $D$ and $B$ amplitudes as functions of $t$ and $v$, where $v=(s-u) /\left(4 m_{N}\right)$, and the Mandelstam variables are given by $s=(p+q)^{2}=\left(p^{\prime}+q^{\prime}\right)^{2}, t=$ $\left(q-q^{\prime}\right)^{2}=\left(p-p^{\prime}\right)^{2}$, and $u=\left(p-q^{\prime}\right)^{2}=\left(p^{\prime}-q\right)^{2}$. They satisfy the identity $s+t+u=2 m_{N}^{2}+2 M_{\pi}^{2}$ with $m_{N}$ and $M_{\pi}$ the physical masses of the nucleon and the pion, respectively.

The amplitudes $X \in\left\{D^{+}, D^{-} / v, B^{+} / v, B^{-}\right\}$are even functions of $v$ and the difference between the full pion-nucleon scattering amplitude and the pseudovector Born term can be expanded around the point $v=t=0[22,23]$ (subthreshold expansion)

$$
X(v, t)=X_{p v}(v, t)+\sum_{i, j=0}^{\infty} x_{i j} v^{2 i} t^{j}
$$

where $X_{p v}(v, t)$ are the pseudovector Born terms and $x \in\left\{d^{+}, d^{-}, b^{+}, b^{-}\right\}$.

The one-particle irreducible tree order contributions to the amplitudes can be parameterized as

$$
\begin{aligned}
& D^{+}=d_{0}^{+}(t, M)+d_{2}^{+}(t, M) v^{2}+d_{4}^{+}(t, M) v^{4}+\cdots \\
& D^{-}=d_{1}^{-}(t, M) v+d_{3}^{-}(t, M) v^{3}+\cdots \\
& B^{+}=b_{1}^{+}(t, M) v+b_{3}^{+}(t, M) v^{3}+\cdots \\
& B^{-}=b_{0}^{-}(t, M)+b_{2}^{-}(t, M) v^{2}+\cdots
\end{aligned}
$$

where $d_{j}^{ \pm}(t, M)$ and $b_{j}^{ \pm}(t, M)$ are Taylor series in $t$ and $M$ - the leading order (LO) term in the chiral expansion of the pion mass. Various contributions to the amplitudes at low energies are organized according to the power counting assigning order $q^{2}$ to $t, q^{1}$ to $v$ and order $q^{1}$ to $M$, where $q$ denotes a small quantity. The amplitudes of Eq. (1.3) are generated by the low-energy effective Lagrangian of corresponding orders. Terms of the effective Lagrangian generating (leading) tree diagrams of order $q^{N}$ are assigned order $N$.

For the purposes of the presented extention of BChPT we re-expand the amplitudes of Eq. (1.3) at $v^{2}=\mu^{2}$ as follows

$$
\begin{aligned}
D^{+} & =d_{0}^{+}(t, M)+v^{2}\left[\tilde{d}_{2}^{+}(t, M)+\tilde{d}_{4}^{+}(t, M)\left(v^{2}-\mu^{2}\right)+\cdots\right] \\
D^{-} & =d_{1}^{-}(t, M) v+v^{3}\left[\tilde{d}_{3}^{-}(t, M)+\tilde{d}_{5}^{-}(t, M)\left(v^{2}-\mu^{2}\right)+\cdots\right] \\
B^{+} & =v\left[\tilde{b}_{1}^{+}(t, M)+b_{3}^{+}(t, M)\left(v^{2}-\mu^{2}\right)+\cdots\right] \\
B^{-} & =\tilde{b}_{0}^{-}(t, M)+\tilde{b}_{2}^{-}(t, M)\left(v^{2}-\mu^{2}\right)+\cdots
\end{aligned}
$$

Note here that the special treatment of $D^{+}$is caused by the fact that $d_{00}^{+}=0$, i.e. we keep at each order the property that $D^{+}=0$ for $t=M=v=0$. Analogously, the fixed value $d_{00}^{-}=1 /\left(2 F^{2}\right)$, where $F$ is the pion decay constant in that limit, causes the special treatment of $D^{-}$. The series of Eq. (1.4) can be generated by an effective Lagrangian with the same structures as contained in the standard effective Lagrangian, however, where the terms are re-arranged according to new power counting. In particular, considering $Q$ as a new small parameter, we count $t \sim Q^{2}, M \sim Q$, and $v^{2}-\mu^{2} \sim Q$. In the Taylor series of $\tilde{d}_{i}^{ \pm}(t, M)$ and $\tilde{b}_{i}^{ \pm}(t, M)$ we keep a finite number of terms, corresponding to the given specified order of accuracy. We assign order $N$ to those terms of the re-arranged effective Lagrangian, i.e. combinations of the chirally invariant structures, which generate contributions 
of order $Q^{N}$ at tree level. At any finite order the re-arranged effective Lagrangian contains a finite number of chirally invariant structures, identical with those of the standard Lagrangian. In particular, each chirally invariant term of the low-energy effective Lagrangian is split into an infinite number of contributions in an infinite number of terms of the re-arranged Lagrangian. The sum of the coefficients of all these infinite number of contributions of the same chirally invariant structure (in terms of growing orders of the re-arranged effective Lagrangian) has to reproduce the coefficient of the corresponding term in the low-energy effective Lagrangian, at least formally. Note here that if one is comparing the low-energy effective Lagrangian without resonances to the re-arranged effective Lagrangian with resonances included as dynamical degrees of freedom, then one needs to remember that low-energy couplings also get contributions from resonances when they are integrated out.

To be more specific, the lowest order terms in $D^{+}$are proportional to $v^{2}, M^{2}$ or $t$, and hence are of order $Q^{0}, Q^{2}$ and $Q^{2}$, respectively. Therefore, terms of the low-energy effective Lagrangian of order $q^{2+2 i}$ giving contributions to $D^{+}$proportional to $v^{2+2 i}$, count as order $Q^{i}$. Terms of order $q^{2 i+2 j+2 k}$ which give contributions proportional to $v^{2 i}\left(M^{2}\right)^{j} t^{k}(j+k \neq 0)$ count as of order $Q^{i+2 j+2 k}$. Here and below by the order of a given structure is meant the lowest order, to which it contributes.

The LO term in $D^{-}$is generated by the covariant derivative part of the standard LO low-energy pion-nucleon Lagrangian $\mathscr{L}_{\pi N}^{(1)}$ [3], which cannot be re-arranged because it generates the undressed propagator of the nucleon. The first subleading terms are proportional to $v^{3}, v M^{2}$ and $v t$ which are of order $Q^{0}, Q^{2}$ and $Q^{2}$, respectively. Therefore, terms of the low-energy effective Lagrangian of order $q^{3+2 i}$, giving contributions to $D^{-}$proportional to $v^{3+2 i}$, count as order $Q^{i}$. Terms of order $q^{1+2 i+2 j+2 k}$ giving contributions proportional to $v^{1+2 i}\left(M^{2}\right)^{j} t^{k}(j+k \neq 0)$ count as order $Q^{i+2 j+2 k}$.

The lowest order terms in $B^{+}$are of order $Q^{0}$, proportional to $v$. Therefore, terms of the lowenergy effective Lagrangian of order $q^{3+2 i}$, giving contributions to $B^{+}$proportional to $v^{1+2 i}$, count as order $Q^{1+i}$. Terms of the order $q^{3+2 i+2 j+2 k}$ giving contributions proportional to $v^{1+2 i}\left(M^{2}\right)^{j} t^{k}$ $(j+k \neq 0)$ count as order $Q^{1+i+2 j+2 k}$. Note here that the amplitudes $B^{ \pm}$are multiplied with $\left[q^{\prime}, q\right]$, which gives two additional orders of the small parameter $q$ in low-energy region and one additional order of $Q$ in the higher-energy region. We assign $Q^{1}$ to the factor $\left[q^{\prime \prime}, q\right]$ according to its contribution to the cross section in the energy region beyond the threshold.

The lowest order terms in $B^{-}$are of order $Q^{0}$, proportional to $v^{0}$. Therefore, terms of the lowenergy effective Lagrangian of order $q^{2+2 i}$, giving contributions to $B^{-}$proportional to $v^{2 i}$, count as order $Q^{1+i}$. Terms of order $q^{2+2 i+2 j+2 k}$, giving contributions to $B^{-}$proportional to $v^{2 i}\left(M^{2}\right)^{j} t^{k}$ $(j+k \neq 0)$ count as order $Q^{1+i+2 j+2 k}$.

To practically construct the $N^{\text {th }}$ order re-arranged Lagrangian using the above power counting for tree-order contributions, first we need to examine all structures of the standard BChPT Lagrangian up to (including) order $2(N+1)+1$ and determine their orders for the higher energy region according to their contributions in the tree-order amplitudes. Next, we need to re-arrange the structures of the low-energy effective Lagrangian in such a way that power series expansions in terms of $v^{2}-\mu^{2}$ appear in tree-order amplitudes. The $k^{\text {th }}$ order re-arranged effective Lagrangian we denote by $\tilde{\mathscr{L}}_{\pi N}^{(k)}$.

The tree diagrams contributing to the $\pi N$ scattering amplitudes at $q^{3}$ order are shown in Fig. 1. Below we specify explicitly the amplitudes corresponding to one-particle irreducible tree-order diagrams which are generated by the standard low-energy effective Lagrangian up-to-including the 
third order [20,21]. For the purpose of the re-arranged theory we also include one forth-order and one fifth-order terms:

$$
\begin{aligned}
D_{\text {tree }}^{+} & =\frac{16 c_{2} m_{N}^{2} v^{2}}{8 F^{2} m^{2}}-\frac{4 c_{1} M^{2}}{F^{2}}+\frac{c_{3}\left(2 M_{\pi}^{2}-t\right)}{F^{2}}+\frac{16 e_{16} v^{4}}{F^{2}}+\cdots, \\
D_{\text {tree }}^{-} & =\frac{v}{2 F^{2}}+\frac{4 d_{3} v^{3}}{F^{2}}+\frac{2 v\left[2 M_{\pi}^{2}\left(2 d_{5}+d_{1}+d_{2}\right)-\left(d_{1}+d_{2}\right) t\right]}{F^{2}}+\frac{f_{x} v^{5}}{F^{2}}+\cdots, \\
B_{\text {tree }}^{+} & =\frac{4\left(d_{14}-d_{15}\right) m_{N} v}{F^{2}}+\cdots, \\
B_{\text {tree }}^{-} & =\frac{1}{2 F^{2}}+\frac{2 c_{4} m_{N}}{F^{2}}+\cdots
\end{aligned}
$$

Here, $m$ and $F$ are the nucleon mass and pion decay constant in the chiral limit, respectively, and the $c_{i}, d_{i}$ and $e_{i}$ are the low-energy constants of the standard effective Lagrangian [21] and $f_{x}$ is a linear combination of coupling constants of the fifth-order effective Lagrangian.

Below we show the new tree-order expressions obtained by re-arranging these terms. Contributions of different orders are put in square brackets and the corresponding orders of the small parameter $Q$ are indicated as subscripts:

$$
\begin{aligned}
D_{\text {tree }}^{+} & =\left[\frac{16 \tilde{c}_{2} v^{2}}{8 F^{2}}\right]_{0}+\left[\frac{16 \tilde{e}_{16} v^{2}\left(v^{2}-\mu^{2}\right)}{8 F^{2}}\right]_{1}+\cdots, \\
D_{\text {tree }}^{-} & =\left[\frac{v}{2 F^{2}}+\frac{4 \tilde{d}_{3} v^{3}}{F^{2}}\right]_{0}+\left[\frac{\tilde{f}_{x} v^{3}\left(v^{2}-\mu^{2}\right)}{F^{2}}\right]_{1}+\cdots, \\
B_{\text {tree }}^{+} & =\left[\frac{4\left(\tilde{d}_{14}-\tilde{d}_{15}\right) m v}{F^{2}}\right]_{0}+\cdots, \\
B_{\text {tree }}^{-} & =\left[\frac{1}{2 F^{2}}+\frac{2 \tilde{c}_{4} m_{N}}{F^{2}}\right]_{0}+\cdots,
\end{aligned}
$$

where we kept only zeroth order terms in the $B^{ \pm}$amplitudes because of the order $Q^{1}$ prefactor $\left[q^{\prime \prime}, q\right]$. The new parameters $\tilde{c}_{i}, \tilde{d}_{i}$ and $\tilde{e}_{i}$ depend on $\mu$ and they are related to the original low-energy constants.

The LO re-arranged effective Lagrangian of the one-nucleon sector generating the leading zeroth order terms in the expansion of $D_{\text {tree }}^{ \pm}$in Eq. (1.6) reads:

$$
\begin{aligned}
\tilde{\mathscr{L}}_{\pi N}^{(0)} & =\bar{\Psi}\left(i \gamma_{\mu} D^{\mu}-m+\frac{1}{2} g_{A} \gamma_{\mu} u^{\mu} \gamma_{5}\right) \Psi-\frac{\tilde{c}_{2}}{4 m^{2}}\left\langle u_{\mu} u_{v}\right\rangle \bar{\Psi}\left(D^{\mu} D^{v}+\text { h.c. }\right) \Psi \\
& +\frac{\tilde{d}_{3}}{12 m^{3}} \bar{\Psi}\left\{\left[u_{\mu},\left[D_{v}, u_{\lambda}\right]\right]\left(D^{\mu} D^{v} D^{\lambda}+\text { sym. }\right)+\text { h.c. }\right\} \Psi .
\end{aligned}
$$

Here, $\Psi$ denotes the nucleon field, $D_{\mu} \Psi=\left(\partial_{\mu}+\Gamma_{\mu}\right) \Psi$ is the covariant derivative and

$$
u^{2}=U, \quad u_{\mu}=i u^{\dagger} \partial_{\mu} U u^{\dagger}, \quad \Gamma_{\mu}=\frac{1}{2}\left[u^{\dagger}, \partial_{\mu} u\right]
$$

where $U$ is a unimodular unitary $(2 \times 2)$ matrix of the Goldstone boson fields. 


\section{Renormalization of loop diagrams}

To renormalize loop diagrams, we generalise the EOMS scheme by moving the normalization point away from the threshold to larger values of the energy. This corresponds to taking the forward-scattering amplitude at some fixed energy in the chiral limit as an input and calculating the expansion around it. Doing so we obtain the complex renormalized parameters of the effective Lagrangian. The power counting of the previous section is also applicable to loop diagrams, provided that the renormalization point is chosen close to the considered energy region. However, the rules of power counting are more complicated for higher energy regions. The orders assigned to the effective Lagrangian cannot be directly translated into the rules for loop diagrams within the new power counting. In particular, to vertices generated by the re-arranged effective Lagrangian we need to assign their corresponding orders according to $q$-counting. Next we draw all loop diagrams with these vertices and recalculate the orders of loop diagrams obtained using $q$-counting (i.e. for low energy region) to those of $Q$-counting (i.e. of high energy region) analogously to tree-order diagrams. This way all loop diagrams are assigned definite orders of a small parameter $Q$. Equipped with these power counting rules we identify those diagrams which have to be included at given order of accuracy.

Un-renormalized loop diagrams violate the power counting. However, all power counting violating parts are polynomial in external momenta and the quark mass and therefore can be absorbed in the redefinition of the parameters of the effective Lagrangian. To demonstrate the EOMS scheme with a sliding scale we consider a simple one-loop integral:

$$
B_{0}\left(p^{2}, M^{2}, m^{2}\right)=\frac{(2 \pi)^{4-n} \mu_{d}^{4-n}}{i \pi^{2}} \int \frac{d^{n} k}{\left[k^{2}-M^{2}+i \delta\right]\left[(p+k)^{2}-m^{2}+i \delta\right]},
$$

where $n$ is the number of space-time dimensions and $\mu_{d}$ is the scale of dimensional regularization. The integral $B_{0}\left(p^{2}, M^{2}, m^{2}\right)$ is of order $q^{1}$ according to the power counting rules. By subtracting the integral at $p^{2}=\mu_{p}^{2}$ this power counting can be satisfied. Note that by $\mu_{p}^{2}=m^{2}+2 m \mu$ the subtraction point $\mu_{p}$ is related to $\mu$ - the subtraction point used later in section 3, provided that $p^{2}$ is identified with the Mandelstam $s$ of pion-nucleon scattering. Calculating the subtraction terms we obtain:

$$
B_{0}^{S T}=-32 \pi^{2} \bar{\lambda}-2 \ln \frac{m}{\mu_{d}}+1+\left(\frac{\mu_{p}^{2}}{m^{2}}-1\right)\left[\ln \left(\frac{\mu_{p}^{2}}{m^{2}}-1\right)-i \pi\right],
$$

where

$$
\bar{\lambda}=\frac{\mu_{d}^{4-n}}{16 \pi^{2}}\left\{\frac{1}{n-4}-\frac{1}{2}\left[\ln (4 \pi)+\Gamma^{\prime}(1)+1\right]\right\} .
$$

The subtracted integral $B_{0}^{R}=B_{0}-B_{0}^{S T}$ is of order $\mathscr{O}(q)$ if $p^{2} \sim \mu_{p}^{2} \sim m^{2}$, and it is of order $\mathscr{O}(Q)$ if $p^{2} \sim \mu_{p}^{2} \gg m^{2}$. This can be easily seen by expanding $B_{0}^{R}$ in $M$ and $p^{2}-\mu_{p}^{2}$.

\section{Application to pion-nucleon scattering at leading one-loop order}

We have applied the EOMS scheme with sliding scale in the low-energy region of $\pi N$ scattering at order $q^{3}$ and compared the results with those of the EOMS scheme. We choose the subtraction scale $\mu$ as a small quantity for low energies and therefore the standard $q$ counting applies for 


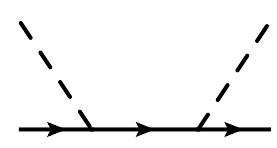

a)

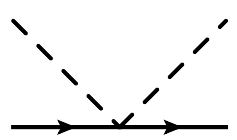

d)

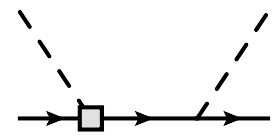

b)

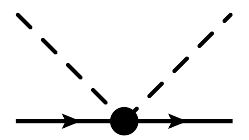

e)

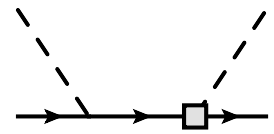

c)

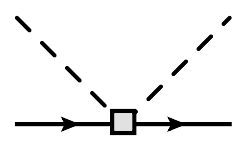

f)

Figure 1: Tree diagrams contributing to the pion-nucleon scattering at order $\mathscr{O}\left(q^{3}\right)$. The solid and dashed lines correspond to the nucleon and the pion, respectively. Crossed diagrams are not shown. Different interaction vertices correspond to different orders.

tree as well as for loop diagrams. The relative values of contributions of different diagrams change for larger values of $\mu$ - some of them become more important than others and the new $Q$-counting applies. According to the above specified rules of $Q$-counting, the loop diagrams of order $q^{3}$ start contributing at order $Q^{0}$ and Loop diagrams of order $q^{4}-$ at order $Q^{1}$. That is, the calculation of this section corresponds to the full $Q^{0}$ calculation of diagrams involving only pions and nucleons if considered at higher energies (and hence for large values of $\mu$ ). For phenomenological applications in the energy region where resonances contribute it is necessary to include them as dynamical degrees of freedom in the effective Lagrangian. For example, for $\pi N$ elastic scattering up to 1.6 $\mathrm{GeV}$, in the $P_{33}$ partial wave we need to include the $\Delta(1232)$ and the $\Delta(1600)$ as explicit degrees of freedom.

The lowest-order pion-nucleon Lagrangian, generating the nucleon propagator and vertices contributing in loop diagrams at $q^{3}$ order, is given by [3]

$$
\mathscr{L}_{\pi N}^{(1)}=\bar{\Psi}\left(i \gamma_{\mu} D^{\mu}-m+\frac{1}{2} g_{A} \gamma_{\mu} u^{\mu} \gamma_{5}\right) \Psi
$$

and the lowest-order $\mathscr{O}\left(q^{2}\right)$ effective mesonic Lagrangian reads [2]

$$
\mathscr{L}_{2}=\frac{F^{2}}{4} \operatorname{Tr}\left(\partial_{\mu} U \partial^{\mu} U^{\dagger}\right)+\frac{F^{2} M^{2}}{4} \operatorname{Tr}\left(U^{\dagger}+U\right) .
$$

The pion-nucleon Lagrangian of second and third orders, needed for our tree diagrams, are specified in Refs. [20, 21]. Figures 1 and 2 contain tree and loop diagrams (respectively), contributing to the pion-nucleon scattering at $q^{3}$ order. We calculated all tree and loop diagrams and subtracted the power counting violating terms from the last. We obtained the subtraction terms by expanding the $D^{ \pm}$amplitudes generated by the loop diagrams in powers of $M, t$ and $v^{2}-\mu^{2}$ up to order $q^{2}$, where we counted $M$ as order $q^{1}$ and $t$ and $v^{2}-\mu^{2}$ as order $q^{2}$. The $B^{ \pm}$are multiplied by $\left[q^{\prime \prime}, q\right]$, which counts as order $q^{2}$, therefore we only need to subtract the terms of zeroth order from them. We checked that all subtraction terms are absorbed by redefining the corresponding coupling constants of the effective Lagrangian. While the subtraction terms are complex for $\mu \neq 0$, we checked that in the $\mu \rightarrow 0$ limit we reprodce the real-valued subtraction terms of the EOMS scheme [24, 25]. Further, following the strategies of Refs. [24, 25], we determined all the involved coupling constants for $\mu=0$ by fitting to the phase shifts of the GWU/SAID group [26] and obtained results 
very similar to those of Refs. [24, 25]. To obtain the coupling constants for $\mu \neq 0$ we used the renormalization group equations with respect to $\mu$.

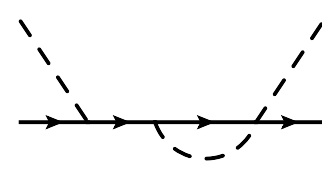

a)

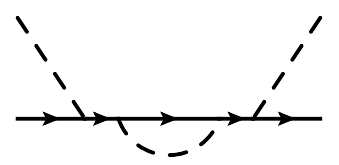

e)

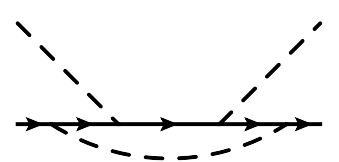

i)

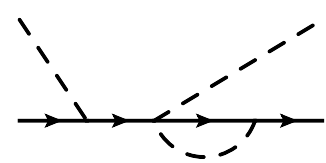

n)

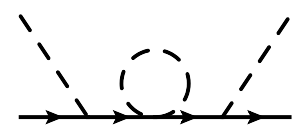

s)

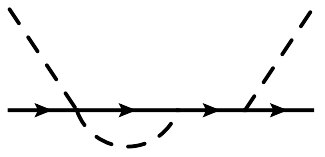

b)

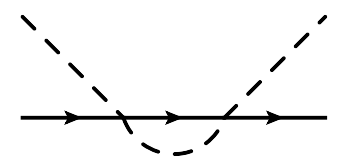

f)

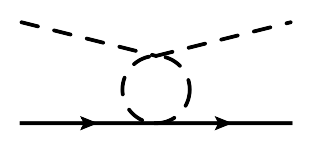

k)

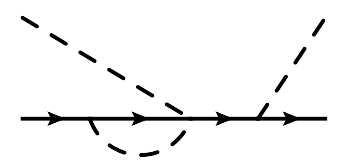

o)

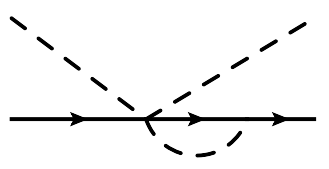

t)

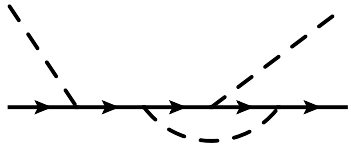

c)

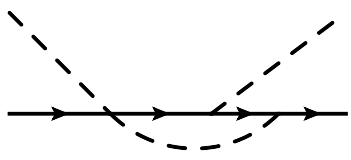

g)

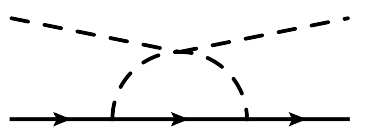

I)

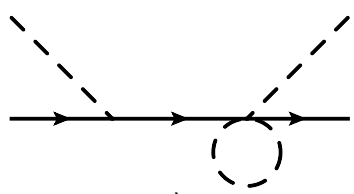

p)

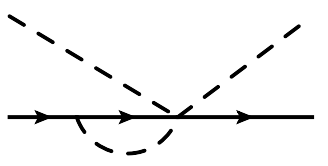

u)

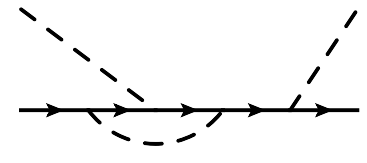

d)

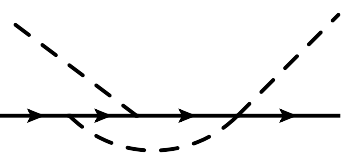

h)

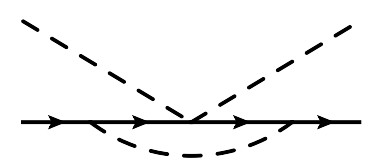

m)

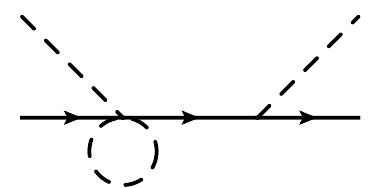

r)

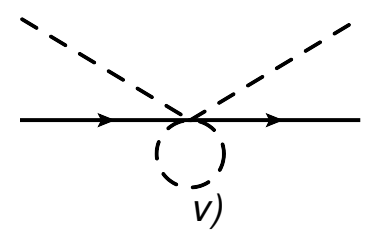

Figure 2: Loop diagrams contributing to the pion-nucleon scattering at order $\mathscr{O}\left(q^{3}\right)$. The solid and dashed lines correspond to the nucleon and the pion, respectively. Crossed diagrams are not shown.

To carry out the renormalization the bare parameters of the effective Lagrangian are expressed in terms of renormalized ones thus generating the main interaction terms and counterterms. We fix these counterterms once, by adjusting them to subtraction terms of the pion-nucleon scattering amplitudes. The same terms of the effective Lagrangian contribute to other processes and hence the same renormalized couplings and counterterms appear. Clearly, it is guaranteed that such an approach respects all underlying symmetries encoded in the effective Lagrangian. We cannot give a general proof that the same counterterms also remove the power counting violating terms from loop diagrams contributing to various related processes, however we expect that this is the case. In any case, as the Ward identities derived from symmetries of the effective Lagrangian are satisfied order-by-order of the expansion around any kinematical point, with certain care by performing subtractions of loop diagrams, it should be possible to respect the symmetries.

\section{Conclusions}

In this contribution we briefly introduced a new approach to BChPT which is applicable for 
processes in the one-nucleon sector at small scattering angles at energies beyond the low-energy regions. Despite the higher energies, the quark structure of hadrons is still not resolved in this kinematical region. For the energies beyond the threshold region contributions of tree-order diagrams have to be re-ordered. This is can be achieved in a systematic and self-consistent way by rearranging the chirally invariant terms of the standard low-energy effective Lagrangian. Resonances, corresponding to the poles of the $S$-matrix in the considered energy region have to be included as explicit degrees of freedom in the effective Lagrangian. Effective field theoretical approach based on such an effective Lagrangian possesses a well defined power counting for tree diagrams. To be able to apply the same power counting also for loop diagrams, we generalised EOMS scheme by using the subtraction at sliding scale. Within this scheme we shifted the renormalization point in the physical region beyond the threshold. The resulting renormalized loop diagrams satisfy a systematic power counting in the neighbourhood of the subtraction point, provided that small scattering angles are considered. However by choosing the renormalization point in the physical region we subtract also the imaginary parts of loop diagrams. This leads to complex renormalized couplings and complex counterterms. Finally we are left with a systematic effective field theoretical approach with a well defined power counting. To achieve a finite accuracy for physical quantities a finite number of tree and loop Feynman diagrams have to be calculated within the considered approach. The suggested framework can also be applied to the pion photo- and electro-production processes as well as Compton scattering and processes involving several pions and/or photons for special kinematics.

\section{Acknowledgments}

Supported in part by Georgian Shota Rustaveli National Science Foundation (grant FR/417/6100/14), DFG (SFB/TR 16, "Subnuclear Structure of Matter"), and the ERC project 259218 NUCLEAREFT.

\section{References}

[1] S. Weinberg, Phenomenological Lagrangians, Physica A 96, 327 (1979).

[2] J. Gasser and H. Leutwyler, Chiral Perturbation Theory To One Loop, Ann. Phys. (N.Y.) 158, 142 (1984).

[3] J. Gasser, M. E. Sainio, and A. Švarc, Nucleons With Chiral Loops, Nucl. Phys. B307, 779 (1988).

[4] E. Jenkins and A. V. Manohar, Baryon Chiral Perturbation Theory Using A Heavy Fermion Lagrangian, Phys. Lett. B 255, 558 (1991); 259, 353 (1991).

[5] V. Bernard, N. Kaiser, J. Kambor, and U.-G. Meißner, Chiral structure of the nucleon, Nucl. Phys. B388, 315 (1992).

[6] V. Bernard, N. Kaiser, and U.-G Meißner, Chiral dynamics in nucleons and nuclei, Int. J. Mod. Phys. E 4, 193 (1995).

[7] H. Tang, A new approach to chiral perturbation theory for matter fields, hep-ph/9607436.

[8] P. J. Ellis and H. B. Tang, Pion nucleon scattering in a new approach to chiral perturbation theory, Phys. Rev. C 57, 3356 (1998). 
[9] T. Becher and H. Leutwyler, Baryon chiral perturbation theory in manifestly Lorentz invariant form, Eur. Phys. J. C 9, 643 (1999).

[10] J. Gegelia and G. Japaridze, Matching heavy particle approach to relativistic theory, Phys. Rev. D 60, 114038 (1999).

[11] J. Gegelia, G. Japaridze and X. Q. Wang, Is Heavy baryon approach necessary?, J. Phys. G 29, 2303 (2003).

[12] T. Fuchs, J. Gegelia, G. Japaridze and S. Scherer, Renormalization of relativistic baryon chiral perturbation theory and power counting, Phys. Rev. D 68, 056005 (2003).

[13] V. Bernard, Chiral Perturbation Theory and Baryon Properties, Prog. Part. Nucl. Phys. 60, 82 (2008).

[14] S. Scherer and M. R. Schindler, A Primer for Chiral Perturbation Theory, Lect. Notes Phys. 830, pp.1 (2012).

[15] E. Epelbaum, J. Gegelia, U.-G. Meißner and De-Liang Yao, Baryon chiral perturbation theory extended beyond the low-energy region, in preparation.

[16] R. G. Stuart, in $Z^{0}$ Physics, ed. J. Tran Thanh Van (Editions Frontieres, Gif-sur-Yvette, 1990), p.41.)

[17] A. Denner, S. Dittmaier, M. Roth and D. Wackeroth, Predictions for all processes $e+e-->4$ fermions + gamma, Nucl. Phys. B560, 33 (1999).

[18] A. Denner and J. N. Lang, The Complex-Mass Scheme and Unitarity in perturbative Quantum Field Theory, arXiv:1406.6280 [hep-ph].

[19] T. Bauer, J. Gegelia, G. Japaridze and S. Scherer, Complex-mass scheme and perturbative unitarity, Int. J. Mod. Phys. A 27, 1250178 (2012).

[20] N. Fettes, U.-G. Meißner and S. Steininger, Pion - nucleon scattering in chiral perturbation theory. 1. Isospin symmetric case, Nucl. Phys. A 640, 199 (1998).

[21] N. Fettes, U.-G. Meißner, M. Mojžiš, and S. Steininger, The chiral effective pion nucleon Lagrangian of order p**4, Ann. Phys. (N.Y.) 283, 273 (2000); 288, 249 (2001).

[22] G. Höhler, in: H. Schopper (Ed.), Landolt-Börnstein, Vol. 9b2, Springer, Berlin, 1983.

[23] T. Becher and H. Leutwyler, Low energy analysis of pi $N \longrightarrow$ pi N, JHEP 0106, 017 (2001).

[24] J. M. Alarcon, J. Martin Camalich and J. A. Oller, Improved description of the $\pi N$-scattering phenomenology in covariant baryon chiral perturbation theory, Annals Phys. 336, 413 (2013).

[25] Y. -H. Chen, D. -L. Yao and H. Q. Zheng, Analyses of pion-nucleon elastic scattering amplitudes up to $O\left(p^{4}\right)$ in extended-on-mass-shell subtraction scheme, Phys. Rev. D 87, no. 5, 054019 (2013).

[26] R. A. Arndt, W. J. Briscoe, I. I. Strakovsky and R. L. Workman, Extended partial-wave analysis of piN scattering data, Phys. Rev. C 74, 045205 (2006) [nucl-th/0605082]. The SAID program, http://gwdac.phys.gwu.edu. 